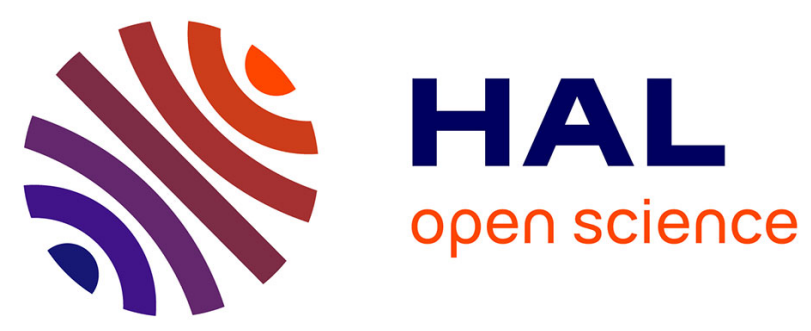

\title{
Closed-loop Control of Silicon Nanotweezers for Improvement of Sensitivity to Mechanical Stiffness Measurement and Bio-Sensing on DNA Molecules
}

Nicolas Lafitte, Yassine Haddab, Yann Le Gorrec, Hervé Guillou, Momoko

Kumemura, Laurent Jalabert, Hiroyuki Fujita, Dominique Collard

\section{To cite this version:}

Nicolas Lafitte, Yassine Haddab, Yann Le Gorrec, Hervé Guillou, Momoko Kumemura, et al.. Closedloop Control of Silicon Nanotweezers for Improvement of Sensitivity to Mechanical Stiffness Measurement and Bio-Sensing on DNA Molecules. IEEE/RSJ International Conference on Intelligent Robots and Systems, IROS'13., Nov 2013, Tokyo, Japan. hal-00912496

\section{HAL Id: hal-00912496 https://hal.science/hal-00912496}

Submitted on 2 Dec 2013

HAL is a multi-disciplinary open access archive for the deposit and dissemination of scientific research documents, whether they are published or not. The documents may come from teaching and research institutions in France or abroad, or from public or private research centers.
L'archive ouverte pluridisciplinaire HAL, est destinée au dépôt et à la diffusion de documents scientifiques de niveau recherche, publiés ou non, émanant des établissements d'enseignement et de recherche français ou étrangers, des laboratoires publics ou privés. 


\title{
Closed-loop control of silicon nanotweezers for improvement of sensitivity to mechanical stiffness measurement and bio-sensing on DNA molecules
}

\author{
Nicolas Lafitte $^{1}$, Yassine Haddab ${ }^{2}$, Yann Le Gorrec ${ }^{2}$, Hervé Guillou ${ }^{1,3}$, Momoko Kumemura ${ }^{1}$, Laurent Jalabert ${ }^{1}$, \\ Hiroyuki Fujita ${ }^{4}$ and Dominique Collard ${ }^{1,4}$
}

\begin{abstract}
In this work we show that implementation of closed loop control to silicon nanotweezers improves the sensitivity of the tool for mechanical characterizations of biological molecules. Micromachined tweezers have already been used for the characterizations of mechanical properties of DNA molecules as well as for the sensing of enzymatic reactions on DNA bundle. However the resolution of the experiments does not allow the sensing on single molecules. Hereafter we show theoretically and experimentally that, reducing the resonance frequency of the system by the implementation of a state feedback, the sensitivity to stiffness variation is enhanced. Such improvement leads to better resolution for detection of enzymatic reactions on DNA.
\end{abstract}

\section{INTRODUCTION}

In the field of molecular biology, a variety of remarkable techniques such as fluidic, electric, magnetic and optical traps have been developed to trap and directly interrogate molecules [1]. These techniques interact mechanically with the molecule and measure the forces related to the structural configuration of the molecule. These have proven the relevance of mechanical characterizations in biology and we have thus developed a MEMS (Micro Electro Mechanical Systems) device which enables these mechanical assays in a systematic manner for diagnostic applications. MEMS are appropriate because they are integrated, monolithic, cheap and can be engineered for specific applications.

The paper is organized as follows. The silicon nanotweezers (SNT), the platform and the sensing method are introduced in the next Section. In Section III, the theory of the closed loop control is developed followed by simulations and experimental results. Especially Section III-B demonstrates experimentally the enhancement of the sensitivity for DNA molecules stiffness. The paper concludes with a discussion on the limitations of the moment (Section IV), and summarizing the capabilities of the developed platform and perspectives (Section V).

\footnotetext{
1 are with the LIMMS/CNRS-IIS (UMI 2820), Institute of Industrial Science, the University of Tokyo, 4-6-1 Komaba, Meguro-ku, Tokyo 1538505, Japan.

2 are with the FEMTO-ST/UFC-ENSMM-UTBM-CNRS, 32 avenue de l'Observatoire, 25044 Besançon, France

3 is with the Institut Néel, CNRS and Université Joseph Fourier, Grenoble, France.

4 are with the Institute of Industrial Science, the University of Tokyo, 4-6-1 Komaba, Meguro-ku, Tokyo 153-8505, Japan.

E-mail: lafittedis.u-tokyo.ac.jp
}

\section{BIO-CHARACTERIZATIONS ON MOLECULES WITH SILICON NANOTWEEZERS (SNT)}

A short description of the device and its operating principle are presented in this Section. The mechanical design, the instrumentation and the sensing method are deeply detailed in [2].

\section{A. Silicon nanotweezers (SNT)}

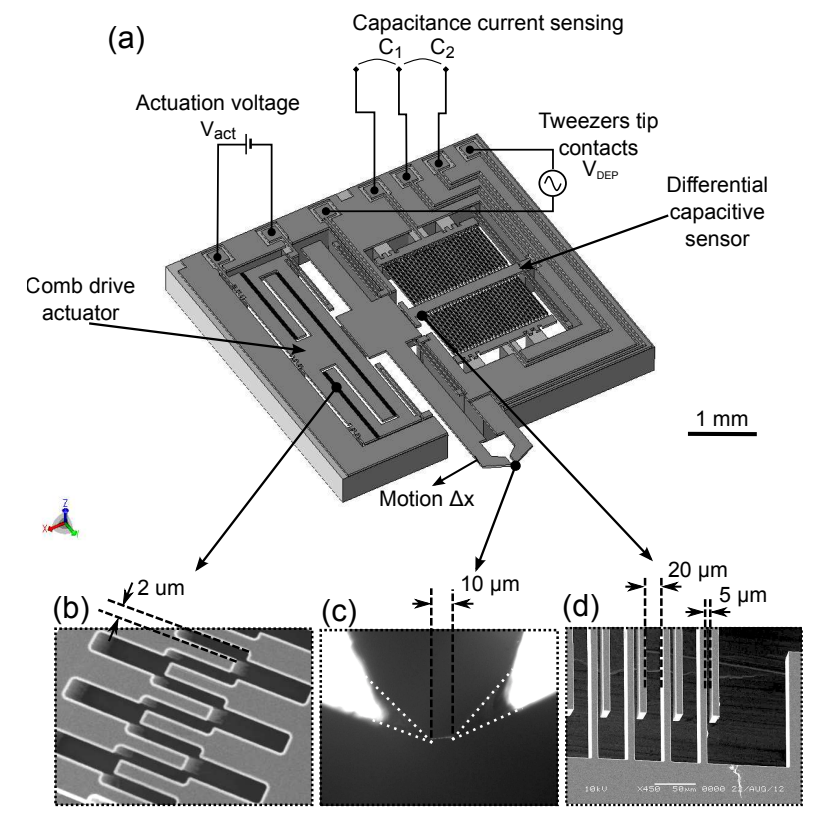

Fig. 1. Silicon nanotweezers (SNT) for bio-electro-mechanical characterizations of molecules bundle. (a) 3D schematic view: the mobile electrode is electrostatically actuated by $V_{\text {act }}$ and the motion displacement $\Delta x$ changes the capacitances $C_{1}$ and $C_{2}$. Chip dimensions: $4.5 \times 4.5 \mathrm{~mm}$. (b) Close view on the electrostatic comb-drive actuator (SEM img). (c) Sharp tips in between a bundle of DNA molecules is trapped (optical microscope img). (d) Close view on the capacitive sensor (SEM img).

The Figure 1 shows a three-dimensional illustration of the device. It consists of two sharp tips that act as electrodes for both DNA trapping by dielectrophoresis and conductivity measurement of DNA molecules [3], [4]. One tip is fixed and the other one moves with an electrostatic actuator. The motion of the electrode is measured using two capacitances with gaps that vary in proportion to the electrode displacement. Therefore with an appropriate electronic read-out (2 current amplifiers and 1 lock-in amplifier), the displacement 
or the velocity of the moving tip is inquired in real-time and continuously [3].

\section{B. Sensing method}

The SNT is driven at its main mechanical resonance which variations are monitored in real-time and enable the quantitative detection of the molecules rigidity. The Figure 2 demonstrates the frequency response of the SNT through the sensor and actuated with a voltage of $1 \mathrm{~V}_{\text {rms }}$.

1) Model: Considering one degree of freedom, we assume that the SNT can be identified as a $2^{\text {nd }}$-order oscillator with $M$ the mass of the moving tip, $k$ the mechanical stiffness of the suspensions and $\nu$ the Stokes viscous losses mainly due to the medium, i.e. air and liquid when tips are immersed in solution. Other modes of resonance arise at higher frequencies which are irrelevant for this study (e.g. at 2.4 and $3 \mathrm{kHz}$ in the Figure 2-a).

2) Characterizations of DNA molecules: The mechanical characteristics of the trapped molecules (rigidity and viscous losses) are measured in real-time by monitoring the resonance frequency of the SNT. When a bundle is trapped in between the tips, the resonance curves change according to the added rigidity $k_{\text {bundle }}$ and the added losses $\nu_{\text {bundle }}$ (Figure 2-b). The mechanical contribution of the sample are extracted from the resonance frequency and amplitude shifts according to the original model of the SNT (Figure 2-c).

3) Resolution: The resolution of the measurements is based on the precision we can measure the peak of the resonance and discriminate shifts in frequency and in amplitude. Resolutions achieved are as low as $10 \mathrm{mHz}$ in frequency and 0.01 in quality factor changes, which corresponds to resolutions in stiffness and in viscous losses of respectively $0.5 \mathrm{mN} \cdot \mathrm{m}^{-1}$ and $0.05 \mu \mathrm{N} \cdot \mathrm{s} \cdot \mathrm{m}^{-1}$. Such resolutions correspond to the sensing of $10 \lambda$-DNA molecules ${ }^{1}$ [5], [6].

These numerical values are obtained after differentiation of the $2^{\text {nd }}$-order oscillator resonance equations:

$$
f_{R}=\frac{1}{2 \pi} \sqrt{\frac{k}{M}} \quad \text { and } \quad Q=\frac{\sqrt{k M}}{\nu}
$$

and from the model parameters (i.e. $M, \nu$ and $k$ ). $f_{R}$ being the frequency and $Q$ the quality factor of the resonance. The derivative $\partial f_{R} / \partial k$ is:

$$
\frac{\partial f_{R}}{\partial k}=\frac{1}{2 \pi} \frac{1}{2 \sqrt{k M}} \text { or } \quad \frac{1}{8 \pi^{2} M f_{R}}
$$

such this sensitivity of the resonance frequency to stiffness variations is $20 \mathrm{~Hz} \cdot \mathrm{N}^{-1} \cdot \mathrm{m}$. These resolutions and this sensitivity limit the method for more relevant experiments at the single-molecule level.

Currently the device is limited by its mechanical structure, especially its stiffness. $k$ is around $30 \mathrm{~N} . \mathrm{m}^{-1}$ and can not be lowered more because of fabrication and manipulation concerns. However the device can be controlled such as to

\footnotetext{
${ }^{1} \lambda$-DNA molecules are a type of DNA molecules we use for our experiments, see Section III-B.3.
}
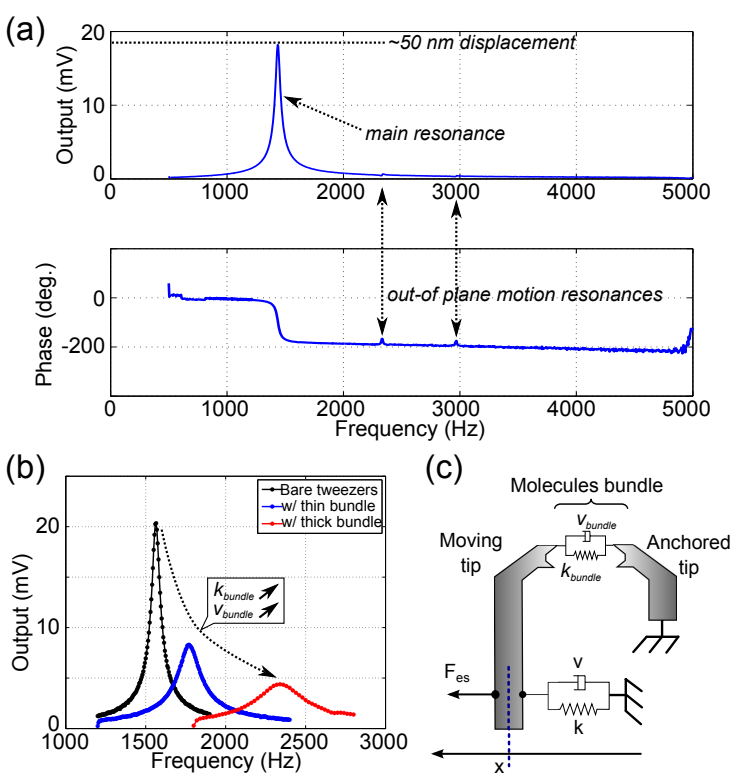

Fig. 2. Model and sensing principle of the SNT. (a) SNT frequency response driven with $1 \mathrm{~V}_{\mathrm{rms}}$ actuation. (b) Variations of the resonance response in air with 2 different DNA bundles. (c) Mechanical model of the system SNT + DNA molecules bundle.

change the characteristics of the system. In the Equation 2, it appears that the variation of $f_{R}$ with respect to $k$ is even more pronounced when $f_{R}$ is small. Sensitivity to stiffness variation can be enhanced by designing a low resonance frequency sensor.

\section{CLOSED-LOOP CONTROL: THEORY, SIMULATIONS AND EXPERIMENTAL RESULTS}

The Section describes the adopted state feedback method, shows simulations and ends with experimental results for the validation.

\section{A. Theory and simulations}

a) Model based control loop method: As aforementioned, the system is represented by an equivalent massspring-damper system shown in Figure 2-c. From Newton's second law we can write:

$$
M \frac{d^{2} x}{d t^{2}}+\nu \frac{d x}{d t}+k x=F_{\mathrm{es}}
$$

where $M$ is the mass of the movable part of the device, $\nu$ is the equivalent viscosity of the system, $k$ is the stiffness of the suspensions and $F_{\mathrm{es}}$ is the electrostatic force applied to the tip through the comb-drive actuator. $k_{\text {bundle }}$ and $\nu_{\text {bundle }}$ are the rigidity and the equivalent of the bundle of molecules are omitted here.

The Equation 3 is so recasted under an equivalent controllable canonical state space representation $(A, B, C)$ where $A \in \mathbb{R}^{2}, B \in \mathbb{R}^{(2,1)}, C \in \mathbb{R}^{(1,2)}$ and $X$ is the state vector 


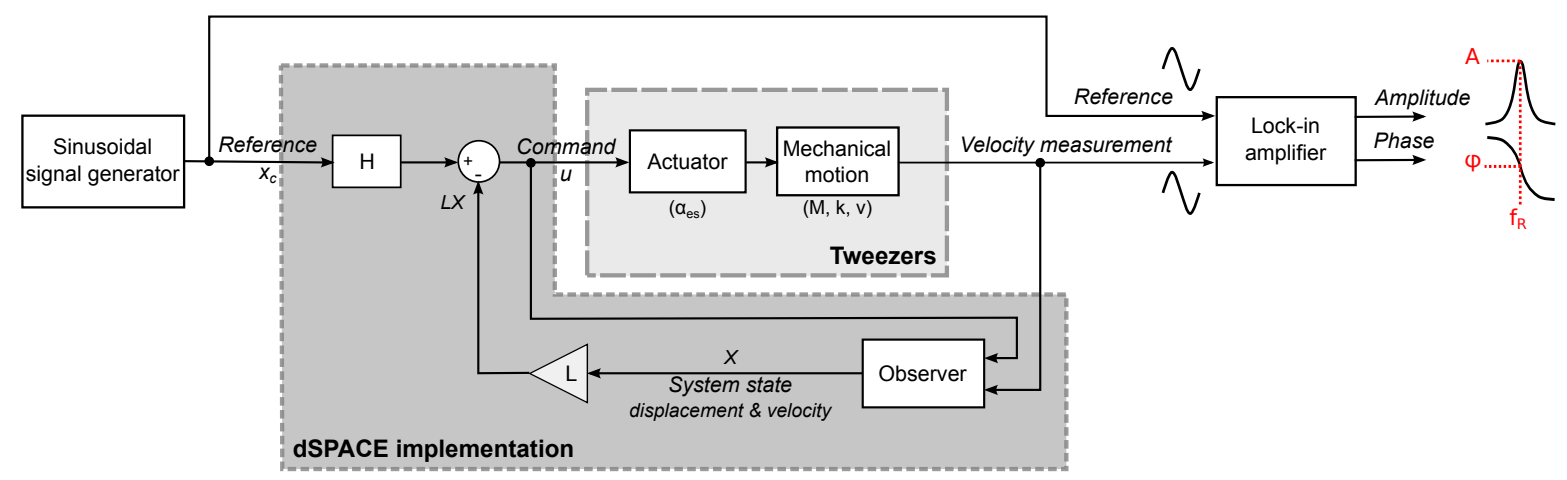

Fig. 3. Feedback control scheme. The Tweezers box includes the mechanical structure of the SNT. The dSPACE implementation box shows the closed-loop elements numerically implemented in the dSPACE prototyping board.

(displacement \& velocity) $X=\left(\begin{array}{l}x \\ \dot{x}\end{array}\right)$.

$$
\dot{X}=\underbrace{\left[\begin{array}{cc}
0 & 1 \\
-\frac{k}{M} & -\frac{\nu}{M}
\end{array}\right]}_{A} X+\underbrace{\left[\begin{array}{c}
0 \\
\frac{1}{M}
\end{array}\right]}_{B} F_{e s}
$$

and

$$
y=\underbrace{\left[\begin{array}{ll}
0 & 1
\end{array}\right]}_{C} X
$$

$y$ is the output vector, i.e. the measurement.

b) State feedback design: The control design strategy is depicted in the flow chart of Figure 3. Accordingly to the motivation, we use a state feedback eigenstructure assignment approach and reduce the resonance frequency of the system through the placement of the poles of the closed-loop system. The drawback of this method is that it is required the implementation of an observer for the reconstruction of the state vector.

From the system under its controllable canonical state space representation $(A, B, C)$ given by Equations 4 and 5 , The state feedback gain vector $L=\left(l_{1}, l_{2}\right) \in \mathbb{R}^{(1,2)}$ is designed to assign the desired closed-loop poles. The closedloop system model becomes:

$$
\dot{X}=\left[\begin{array}{cc}
0 & 1 \\
-\frac{k+l_{1}}{M} & -\frac{\nu+l_{2}}{M}
\end{array}\right] X+\left[\begin{array}{c}
0 \\
1 \\
M
\end{array}\right] F_{e s}
$$

Thus, the closed loop resonance frequency and sensitivity become:

$$
\begin{gathered}
f_{R-c l}=\frac{1}{2 \pi} \sqrt{\frac{\left(k+l_{1}\right)}{M}} \\
\frac{\partial f_{R-c l}}{\partial k}=\frac{1}{4 \pi \sqrt{\left(k+l_{1}\right) M}}=\frac{1}{8 \pi^{2} M f_{R-c l}}
\end{gathered}
$$

such as both values can be controlled by the feedback parameter $l_{1}$. c) Identification of the model parameters: For the development of the feedback method, the model of the device is identified around the operating point over all the experiments are performed. Differences have been pointed out between the theoretical and the final geometrical dimensions of the device after fabrication, leading to parameter uncertainties. Tiny structures such as the mechanical suspensions are more delicate to overetching, changing significantly related parameters as the device stiffness.

The identification is achieved through standard recursive approach with a method of least squares. Responses to small signals around $9 \mathrm{~V}$ offset (i.e. $110 \mathrm{~nm}$ offset) are recorded in open-loop and identified. During the experimentations 2 SNT from the same wafer of fabrication have been used and the identified model parameters for both are summarized in the Table I.

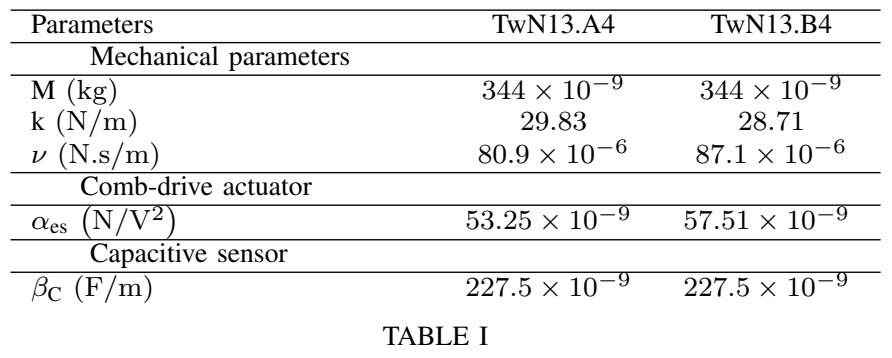

IDENTIFIED PARAMETER VALUES OF THE 2 SILICON NANOTWEEZERS USED IN THESE EXPERIMENTS.

d) Simulations: With the parameters identified in the Table I, the feedback system has been first implemented and tested under Matlab/Simulink without observer (i.e. all the state variables are supposed to be measured).

The Figure 4 shows the resonance frequency shifts of open-loop and closed-loop systems undergoing a stiffness variations from -1 to $1 \mathrm{~N} / \mathrm{m}$. The closed-loop systems with a resonance frequency reduction factor set at $n=1.2$ and $n=2$ have respectively 1.2 and 2 times more important shifts than the open-loop system. Indeed, as theoretically expected, the sensitivities of the closed-loop system are $n$ times enhance.

In the second instance, we design and simulate a Luen- 


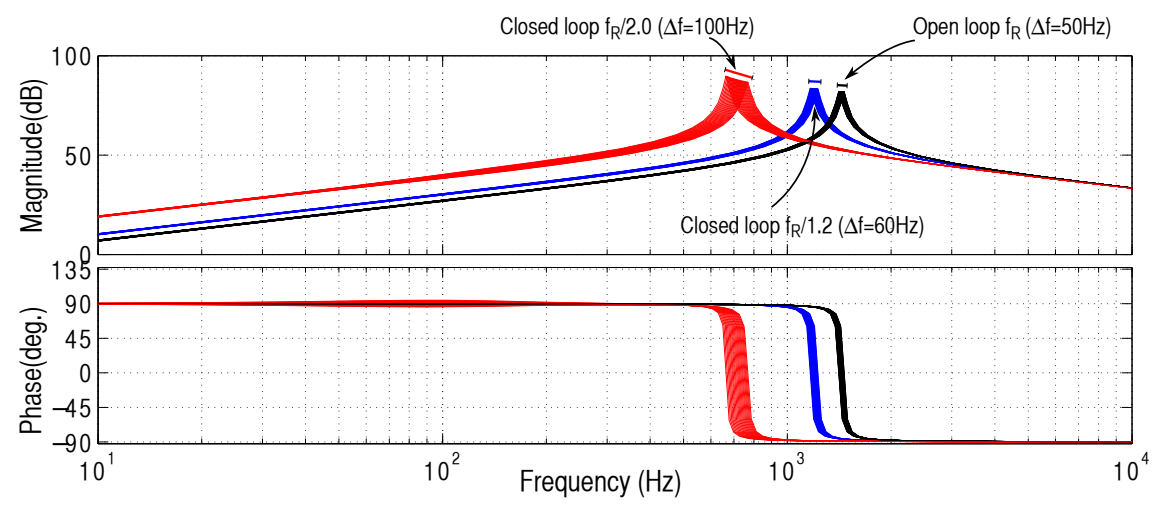

Fig. 4. Simulation results. In black line, the tweezer transfer function. In blue and red lines, the implemented closed-loop system transfer function with respectively $n=1.2$ and $n=2.0$. Both systems undergo a variation of $k$ the system stiffness. $k$ varies from -1 to $1 \mathrm{~N} / \mathrm{m}$ by $0.1 \mathrm{~N} / \mathrm{m}$ steps.

berger observer for the real implementation of the feedback. The design of the observer is not discussed in this paper but the main objective is to reconstruct the system state without altering the closed-loop sensitivity or amplifying the measurement noise. More simulations demonstrate that the overall parametric sensitivity of the system depends on the observer dynamics. In the following experimental part, the observer is specifically designed in such a way that the closed-loop performances are not degraded.

\section{B. Experimental sensing results}

In order to validate the theoretical results, here we aim to conduct different experiments :

- with an added stiffness by contact with a cantilever,

- with an added mass by coating an additional layer of photoresist on the SNT's tip,

- and, finally, characterizing DNA molecules rigidity.

The feedback controller and the observer are implemented in a dSPACE's prototyping board. The Figure 5 shows the results of the three experiment cases. All the responses are fitted with a $2^{\text {nd }}$-order oscillator resonance curve in order to extract the resonance frequency.

1) Added stiffness: First measurements are performed causing variations of the stiffness parameters (Figure 5-A). In order to change the stiffness of the system, a cantilever of calibrated stiffness is put in contact with the SNT's tip. Two experiments are performed with two cantilevers adding different stiffness, respectively 0.3 and $0.42 \mathrm{~N} / \mathrm{m}$ (with $\pm 0.15 \mathrm{~N} . \mathrm{m}^{-1}$ manufacturer tolerance).

In Figure 5-A1, three curves are plotted standing for the experiments 1) without cantilever contact, 2) with the first cantilever of $0.3 \mathrm{~N} / \mathrm{m}$ and 3 ) with the second cantilever of $0.42 \mathrm{~N} / \mathrm{m}$. For each curve/configuration, 7 points plot the resonance frequency of the open loop system $(n=0)$ and for 6 closed-loop systems with different $n$ from 1.1 to 2.1. As expected, it appears that in all the cases the frequency increases due to the stiffness of the cantilevers.

For comparison, Figure 5-A4 plots the shifts in frequency between configurations with and without cantilever contact, for the two cantilevers and for the different closed loop systems. Both curves show a linear increase of the shift in frequency with the reduction factor $n$, expressing an increase of the sensitivity to stiffness variations

However the improvements are more important than expected. First in open-loop, the shifts of frequency are +9.2 and $+15.3 \mathrm{~Hz}$ respectively for stiffness of 0.3 and $0.42 \mathrm{~N} / \mathrm{m}$, instead of +6.0 and $+8.4 \mathrm{~Hz}$ according to Equation 2 . Moreover the improvement of the sensitivity is, for example, $\times 3.5$ instead of $\times 2.1$ in the case of $n=2.1$. The shifts and the improvement are 1.7 times more important than predicted.

2) With mass variations: Another evaluation of the closed loop control is to test the effect when the mass changes (Figure 5-B). In order to add mass to the system, a small layer of photoresist is deposited on the mobile tip. We dip it into a droplet of photoresist (Shipley S1805) prepared on a glass, retrieved and wait until all solvent has evaporated (Figure 5-B3). The remaining dried layer of photoresist increase the inertial mass of the system. The operation is repeated in a second time for a second experiment. This method has the advantage to be simple and efficient but the quantity of photoresist is unknown.

As for the cantilevers, the performances are compared between open loop and closed loop systems with different reduction factor $n$. The Figure 5-B1 shows the resonance frequency of the systems for experiment without additional mass and with the two added layers. As expected in openloop configuration, the resonance frequency decreased first of $-2.3 \mathrm{~Hz}$ and then of $-7.7 \mathrm{~Hz}$ because of the increased inertia. From Equation 1, the added mass is deduced to be $1.1 \mu \mathrm{g}$ after the $1^{\text {st }}$ deposition and $3.7 \mu \mathrm{g}$ after the $2^{\text {nd }}$ deposition.

The Figure 5-B4 compares the shifts in frequency between the systems with and without additional mass and for both added mass. It appears in both cases that the variations are first negative and tend to 0 , then becomes positive and increases for large reduction of the resonance frequency. By theory, it is expected that the negative variation shifts decrease until 0 . Furthermore, it is hard to conceive that an increased of the mass tend to cause positive variation of the 

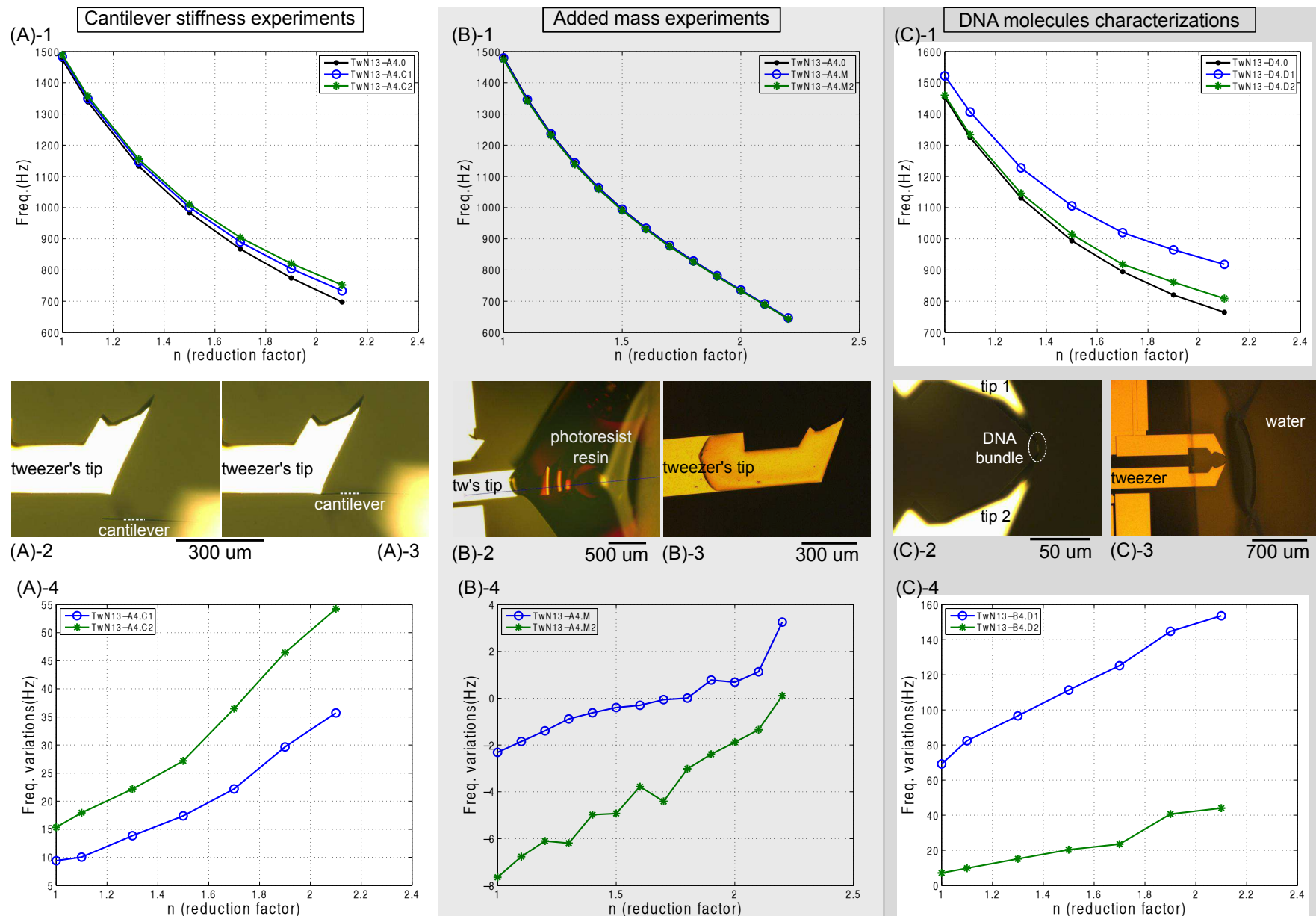

Fig. 5. Experimental results comparing the sensitivities of different closed-loop systems (A) when a stiffness is added, (B) when a mass is added and (C) for the mechanical characterizations of DNA molecules bundle. Resonance frequencies of closed-loop systems are designed with different reduction factor $n$ of the original resonance frequency $\left(f_{R} \simeq 1480 \mathrm{~Hz}\right) . n=1.0$ corresponds to the open-loop configuration.

(A) Cantilever experiments: (A)-1 Resonance frequencies of systems with different reduction factor $n$ from 1.1 until 2.1 with 0.2 steps. The black curve is the bare tweezers systems without cantilever contact. The blue curve is with the cantilever of $0.3 \mathrm{~N} / \mathrm{m}$ stiffness in contact with the tweezers tips (Figure (A)-3). The green curve is with a shorter and stiffer cantilever $(0.42 \mathrm{~N} / \mathrm{m})$. (A)-2 \& -3 Microscopy images of the cantilever chip respectively in approach of the tweezer's tip and in contact with the tweezer's tip. (A)-4 Resonance frequency shifts due to the added stiffness.

(B) Added mass experiments: (B)-1 Resonance frequencies of systems with different reduction factor $n$ from 1.1 until 2.2 with 0.1 steps. The black curve is the bare tweezers system. The blue curve is with an added layer of photoresist on the tweezers tips (Figure (B)-3). The green curve is with one more additional layer of photoresist coated at the end of tip. (B)-2 \& -3 Microscopy image of the immersion of the tip in S1818 photoresist and of the resulting remaining photoresist layer on the tip. (B)-4 Resonance frequency shifts due to the added mass

(C) $\lambda$-DNA characterization experiments: (C)-1 Resonance frequencies of systems with different reduction factor $n$ from 1.1 until 2.1 with 0.2 steps. The black curve is the bare tweezers systems. The blue curve is with an small trapped $\lambda$-DNA molecule bundle characterized in air (Figure (C)-2). The green curve is with the same molecule bundle characterized very close $(<100 \mu \mathrm{m})$ to a humidity source in order to soften the bundle (Figure (C)-3). (C)-2 Photo of the bundle of $\lambda$-DNA molecules. (C)-3 Photo of the setup softening the DNA bundle because of the close vicinity of water. (C)-4 Resonance frequency shifts due to the DNA bundle.

frequency such as demonstrated. This leads us to understand the cause of this behavior and probably to improve the model and its parameters. This point is considered in the following discussion Section.

3) DNA characterizations: Finally characterizations of DNA molecule bundle have been performed (Figure 5-C). The goal being to characterize, as for the cantilevers, the rigidity of DNA molecules. $\lambda$-DNA molecules (which are DNA molecules of $16 \mu \mathrm{m}$ length of the bacteriophage lambda) are trapped from a DNA solution droplet combining dielectrophoresis [5], [7] and combing methods [8].

The bundle is first characterized in air under biological room conditions (at $22^{\circ} \mathrm{C}$ and with $30 \%$ of humidity). Afterwards the bundle is softened by bringing the bundle at less than $100 \mu \mathrm{m}$ of a $50 \mu \mathrm{L}$ water droplet (Figure 5-C3), and the bundle is tested again. The humidity at this level is not measured.

The Figure 5-C1 shows the characterizations of the bundle rigidity in open loop and closed-loop configurations. In openloop configuration, the resonance frequency increased of $+69.2 \mathrm{~Hz}$ in dry condition and of $+7.1 \mathrm{~Hz}$ in wet condition; such as, from Equation 2, the rigidity of the DNA bundle are deduced to be respectively 2.77 and $0.28 \mathrm{~N} / \mathrm{m}$.

Finally the last Figure 5-C4 demonstrates the shifts in frequency caused by the rigidity of the bundle. The sensi- 
tivity of the resonance frequency to stiffness variations are significantly improved by reducing the resonance frequency in closed loop. Once again the method shows a linear enhancement of the sensitivity upon the reduction factor $n$. The enhancement is again more important than theory predicts and is discussed in the next Section.

\section{DISCUSSION}

In the previous Section, experimental results demonstrate the fulfillment of the initial motivation for this work by the control of the SNT. The enhancement of the sensitivity for more relevant experimentations has been achieved. However, as it has been pointed out, the improvement is more important than theory and simulations predict. Moreover the method is currently limited to a reduction factor of $n=2.2$; further, the closed loop system goes to an unstable behavior (i.e. to saturation or to oscillations). Especially two points are under investigation and discussed here: the relevance of the model and the identification of the model parameters.

The identification of the parameters is a delicate and essential step for the modeling and the development of the feedback. On the one hand, the dimensions and the shape of the smallest parts of the device such as the width of the mechanical suspensions (which are $12 \mu \mathrm{m}$ wide, $30 \mu \mathrm{m}$ thick, and $1 \mathrm{~mm}$ long) are highly dependent on the fabrication. On the other hand, parameters are many and hard to characterize precisely and independently one after one. In this work, the mass $M$ of the movable part have been considered to be a reference being close to the theoretical value. However according to the experiment on sensitivity to added mass, the set of parameters is not yet accurately identified and parameter identification should be improved.

Besides, limitations in the performances may arise from the model of the system. The mechanical motion of the SNT fits accurately with a $2^{\text {nd }}$-order oscillator model and other modes of motion are out of the bandwidth. Nevertheless, in the current instrumentation, the current pre-amplifiers show a low-pass filter behavior with a cut-off frequency $(\sim 2 \mathrm{kHz})$ close to the dynamic of the SNT. Moreover Analog-toDigital and Digital-to-Analog Converters associated with the dSPACE prototyping board bring non-negligible delays to the loop. Both behaviors has been for the moment, considered as a delay and approximated with a Padé model [2].

Work on a more accurate model is on the way on and may lead to a complete validation of the method.

\section{CONCLUSIONS}

A state feedback control has been applied to silicon nanotweezers in order to improve characterizations of biological molecules. Sensing with micromachined tweezers enables systematic and routine experimentations on biological samples such as DNA molecules but, in addition, the sensitivity has been improved by control strategy toward single molecule resolution. The implementation of a feedback control achieved to reduce the resonance frequency and emulate a more compliant (i.e. low stiffness) sensor which increase the sensitivity of the tool to detect rigidity of the molecules.

The method have been illustrated with three cases of experiments: 1) characterizing the rigidity of silicon cantilevers, 2) characterizing the mass of extra deposited layer and finally 3) characterizing DNA molecules bundle rigidity. Before the work, the resolution was of 10 molecules of $\lambda$-DNA. This work allowed experimentally to improve this value by 3.5 and bring the resolution close to the single molecule.

This approach paves the way for diagnostic analysis with micromachined silicon nanotweezers and with single molecule resolution.

\section{ACKNOWLEDGMENT}

The photolithography masks were fabricated with the 8inch EB writer F5112+VD01 donated by Advantest Corporation to the VLSI Design and Education Center (VDEC, the University of Tokyo).

\section{REFERENCES}

[1] C. Bustamante, J. C. Macosko, and G. J. Wuite, "Grabbing the cat by the tail: manipulating molecules one by one." Nature reviews. Molecular cell biology, vol. 1, no. 2, pp. 130-136, Nov. 2000.

[2] N. Lafitte, "Modeling and control of MEMS tweezers for the characterizations of enzymatic reactions on DNA molecules," Ph.D. dissertation, FEMTO-ST/LIMMS, Apr. 2012.

[3] C. Yamahata, D. Collard, B. Legrand, T. Takekawa, M. Kumemura, G. Hashiguchi, and H. Fujita, "Silicon Nanotweezers With Subnanometer Resolution for the Micromanipulation of Biomolecules," Microelectromechanical Systems, Journal of, vol. 17, no. 3, pp. 623631, 2008.

[4] C. Yamahata, D. Collard, T. Takekawa, M. Kumemura, G. Hashiguchi, and H. Fujita, "Humidity dependence of charge transport through DNA revealed by silicon-based nanotweezers manipulation," Biophysical Journal, vol. 94, no. 1, pp. 63-70, 2008.

[5] N. Lafitte, M. Kumemura, M. Nagai, L. Jalabert, D. Collard, and H. Fujita, "An open microfluidic device with active valves for accurate trapping of DNA by silicon nanotweezers," in 14th Int Conf. on Miniaturized Systems for Chemistry and Life Sciences, MicroTAS2010, 2010, pp. 1865-1867.

[6] N. Lafitte, M. Kumemura, L. Jalabert, D. Collard, and H. Fujita, "RealTime sensing of molecule binding on DNA with silicon nanotweezers," in 15th Int Conf. on Miniaturized Systems for Chemistry and Life Sciences, MicroTAS2011, 2011, pp. 389-372.

[7] M. Kumemura, D. Collard, C. Yamahata, N. Sakaki, G. Hashiguchi, and H. Fujita, "Single DNA molecule isolation and trapping in a microfluidic device," ChemPhysChem, vol. 8, no. 12, pp. 1875-1880, 2007.

[8] J. van Mameren, E. J. G. Peterman, and G. J. L. Wuite, "See me, feel me: methods to concurrently visualize and manipulate single DNA molecules and associated proteins," Nucleic Acids Research, vol. 36, no. 13 , pp. $4381-4389$, Aug. 2008. 